\title{
HEPATITIS C VIRUS INFECTION
}

natuire DISEASE REVIEWS PRIMERS

$\rightarrow$ Hepatitis C virus (HCV) is a hepatotropic RNA virus. Following acute infection, the majority of patients develop chronic liver inflammation that can result in cirrhosis and hepatocellular carcinoma. HCV infection is the leading indication for liver transplantation in many parts of the world.

\section{EPIDEMIOLOGY}

The global prevalence of HCV infection, based on the detection of viral RNA in the blood, has been estimated at $\sim 1 \%$, which corresponds to $\sim 80$ million infections. The age distribution of infection varies across countries. The average age of infected individuals is considerably lower in countries where injection drug use is an ongoing risk factor ( $\sim 35$ years) than in countries where iatrogenic infections (that is, infections due to a medical procedure) are the main cause ( 50-60 years) In addition, HCV ( 50-60 years). In addition, HCV genotypes - seven of which have been detected thus far - show geographical variation.

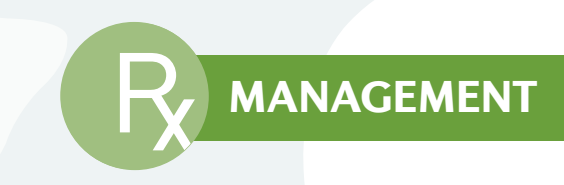

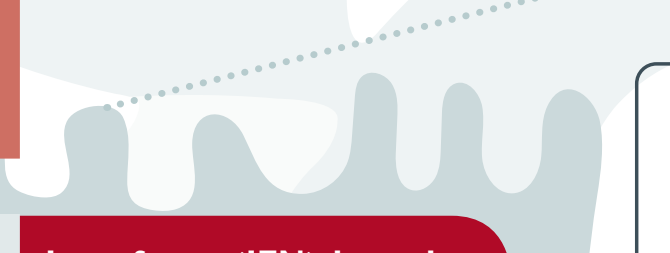

Interferon (IFN)-based treatment used to be common practice, but cure rates were suboptimal and adverse suboptimal and adverse effects were common. In some parts of the
world, including Asia, world, including Asia,
these regimens are still being used.

(2)

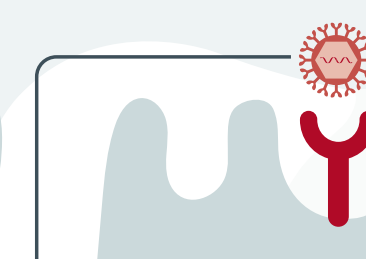

(3)

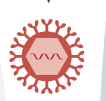

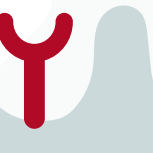

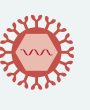

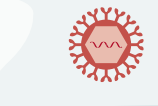

.

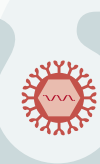

(3)

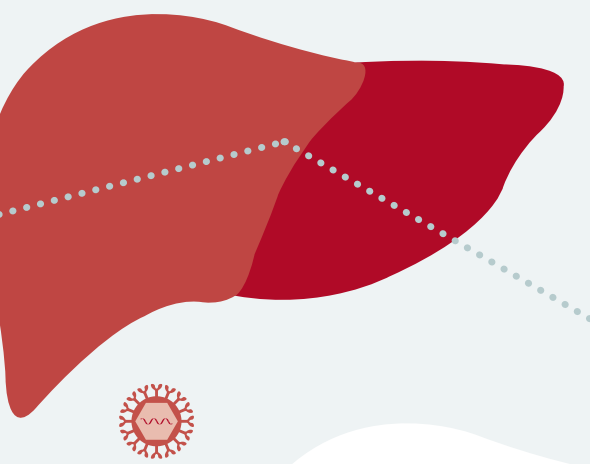

For the Primer, visit do: $10.1038 /$ nrdp.2017.6

\section{DIAGNOSIS}

HCV infection is often asymptomatic, until the liver damage has progressed. Diagnostic, screening and monitoring procedures include HCV antibody testing, HCV RNA measurement, viral genotype and subtype determination and the assessment of resistance-associated substitutions, which are genetic substitutions that make the virus less susceptible

$45-85 \%$ to commonly used DAAs.

\section{$45-85 \%$}

individuals are

unaware of the ir condition.

PREVENTION

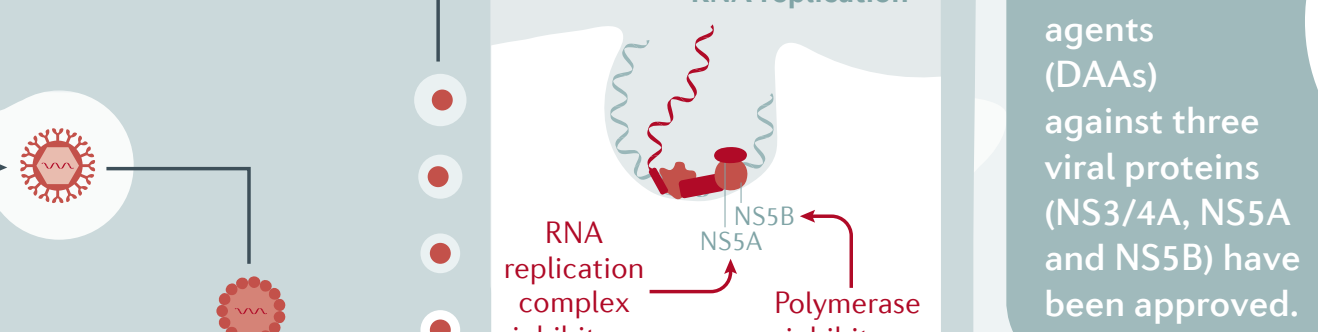
Polymerase
inhibitors $50-60 \%$ of $\mathrm{HCV}$ Europe and the United States are due drug use.
drute injectior

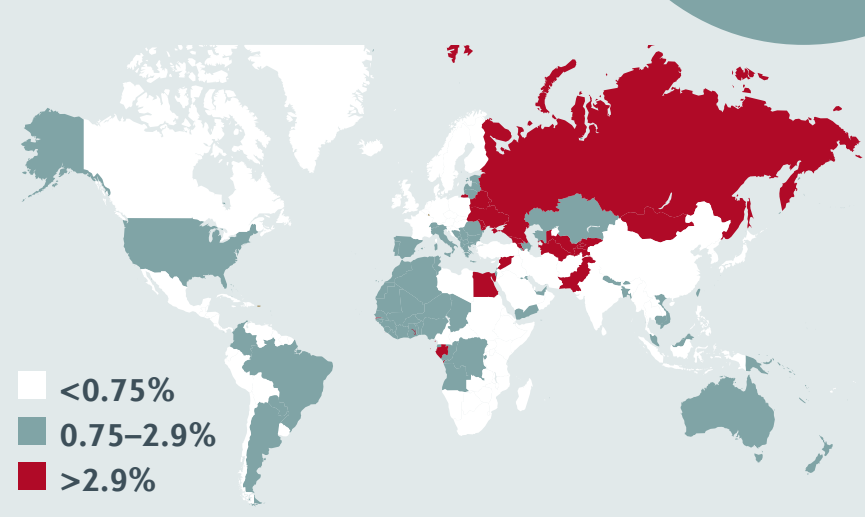
Worldwide prevalence varies considerably,
with the highest prevalence in countries with with the highest prevalence in countries with past or present history of iatrogenic infectior prevalence of $>5 \%$ in the adult population. complex

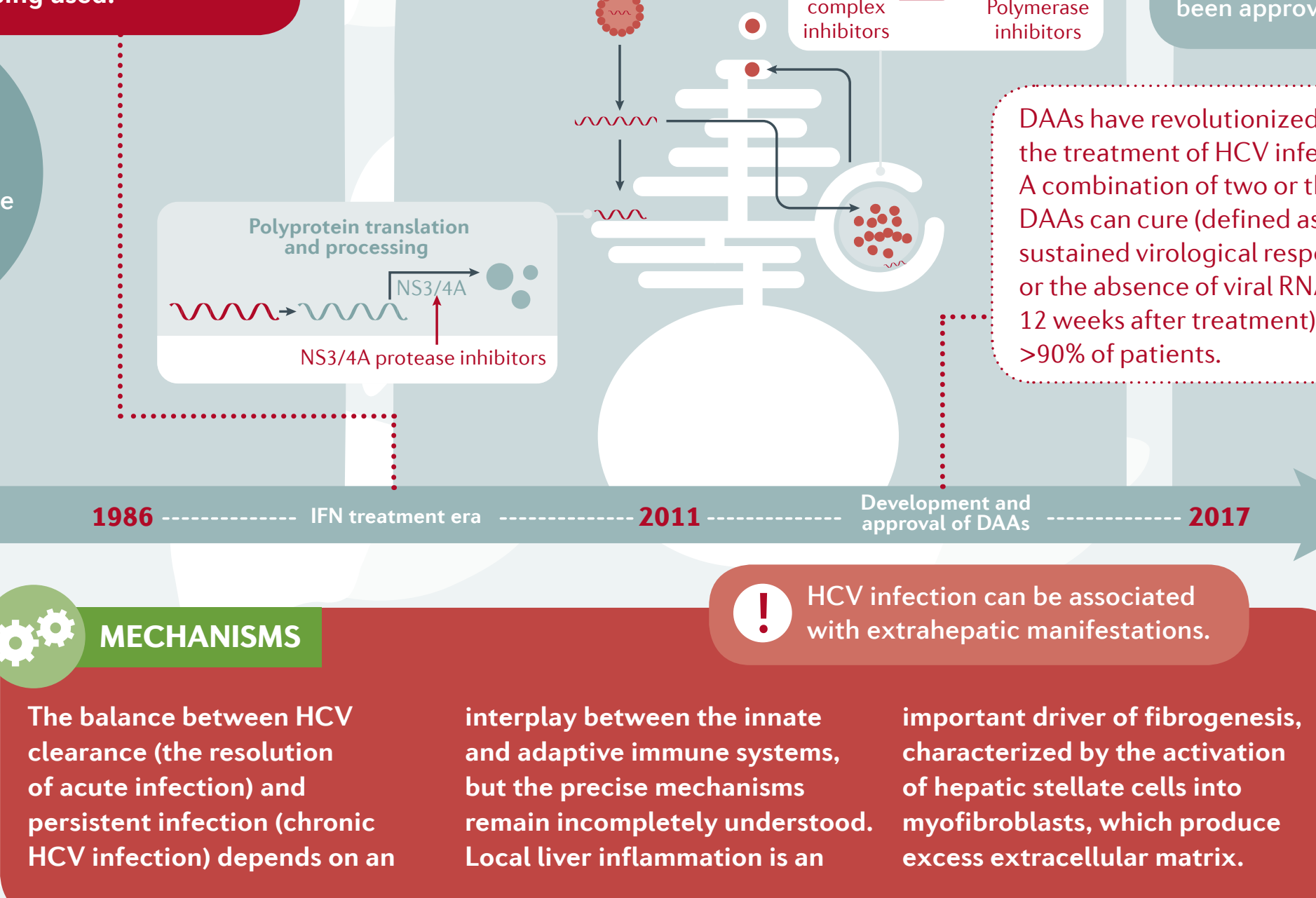

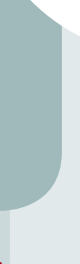

$$
\text { to in }
$$

throinly transmitted

through percutaneous exposure Causes of iatrogenic infections range from blood transfusions or administration of clotting factors (prior to screening that started in the 1990s) to reuse of contaminated materials. Prevention is aimed at minimizing the risk of transmission: avoid direct exposure to blood; do not share needles or personal care items; only use licensed tattoo and piercing parlours; and avoid risky sexual activities. Screening of individuals at increased risk is recommended.

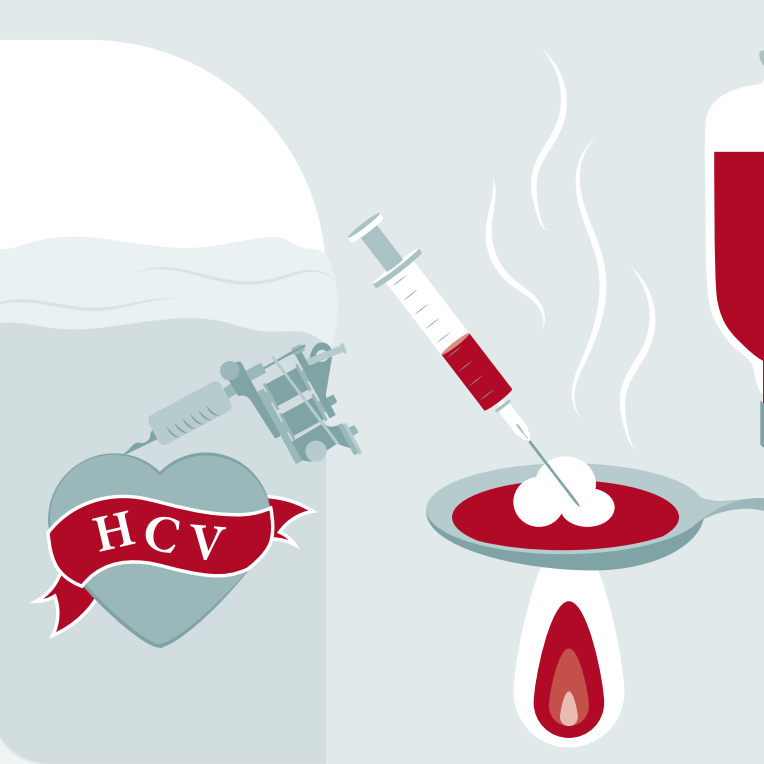

